Case Report

\title{
MYC Amplification in Angiosarcoma Arising from an Arteriovenous Graft Site
}

\author{
Kristen M. Paral, ${ }^{1}$ Gordana Raca, ${ }^{2}$ and Thomas Krausz ${ }^{3}$ \\ ${ }^{1}$ Department of Medicine, Section of Dermatology, University of Chicago, 5841 S Maryland Avenue, MC 5067, Chicago, IL 60637, USA \\ ${ }^{2}$ Department of Medicine, Section of Hematology/Oncology, University of Chicago, 5841 S Maryland Avenue, MC 6092, \\ Chicago, IL 60637, USA \\ ${ }^{3}$ Department of Pathology, University of Chicago, 5841 S Maryland Avenue, MC 6161, Chicago, IL 60637, USA
}

Correspondence should be addressed to Kristen M. Paral; kristen.paral@uchospitals.edu

Received 1 August 2015; Revised 29 October 2015; Accepted 8 November 2015

Academic Editor: Khin Thway

Copyright (C) 2015 Kristen M. Paral et al. This is an open access article distributed under the Creative Commons Attribution License, which permits unrestricted use, distribution, and reproduction in any medium, provided the original work is properly cited.

\begin{abstract}
Angiosarcoma arising in association with an arteriovenous graft (AVG) or fistula is a unique clinicopathologic scenario that appears to be gaining recognition in the literature. Among reported cases, none has described high-level MYC gene amplification, a genetic aberration that is increasingly unifying the various clinicopathologic subdivisions of angiosarcoma. We therefore report the $M Y C$ gene status in a case of angiosarcoma arising at an AVG site.
\end{abstract}

\section{Introduction}

Certain predisposing conditions have been clearly associated with angiosarcoma, including (i) radiation exposure, such as ultraviolet light in skin cases and therapeutic irradiation in breast cases; (ii) longstanding lymphedema (i.e., StewartTreves syndrome); and (iii) exogenous toxins (e.g., vinyl chloride, thorotrast, and arsenic) in liver cases [1]. Angiosarcoma associated with AVG, however, has not yet achieved the same level of recognition as the aforementioned scenarios. Despite a steady trickle of such cases into the literature over the recent decades [2-11], few authoritative sources acknowledge its existence [12].

An AVG or fistula is an artificial connection between an artery and a vein created with synthetic materials such as polytetrafluoroethylene (PTFE) or Dacron [13]. Implanted foreign material has been linked to the development of sarcoma for many decades [14]. Most cases of angiosarcoma associated with an AVG have been linked to immunosuppression, although other etiological factors have been suggested, such as the alteration of blood flow [2,11]. Minimal attention has been devoted to the role of genetic aberrations in these cases.

Recent findings of frequent high-level MYC gene amplification in angiosarcomas suggest a genetic basis for the disease [15]. The MYC gene is a potent oncogene that is amplified in various clinicopathologic subdivisions of angiosarcoma, including secondary (postirradiation and lymphedema-associated) and a subset of primary angiosarcomas (breast, skin, and bone) [15-19]. MYC amplification status has not yet been described in the unusual setting of AVG-associated angiosarcoma; we therefore report MYC amplification status in one such case.

\section{Case Report}

A 63-year-old Hispanic man presented in 2013 for evaluation of left arm pain in an area where an AVG had been placed 15 years previously (a brachiocephalic "loop graft" with PTFE). The AVG had not been in use for eight years owing to a kidney transplant in 2005. Prior to the transplant, the patient required regular hemodialysis due to end-stage renal disease from chronic hypertension and diabetes.

Physical examination revealed swelling and tenderness of the AVG site, which was attributed to probable thrombosis. This was pursued by angiogram, which was interpreted as a thrombosed pseudoaneurysm at the site. The angiogram also disclosed an unexpected finding: a destructive mass involving the elbow. Further imaging with MRI studies revealed a $20 \mathrm{~cm}$ mass encircling the elbow and tracking distally into 


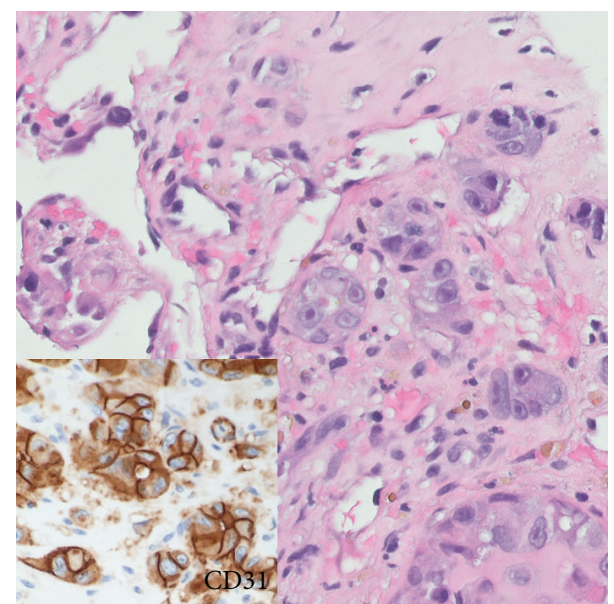

Figure 1: Microscopic examination, biopsy material. The conspicuous clusters of malignant epithelioid cells dominate the picture and could give rise to a broad differential diagnosis that includes carcinoma and melanoma. The poorly formed vascular channels in the background could easily be overlooked. Immunohistochemistry was key (inset).

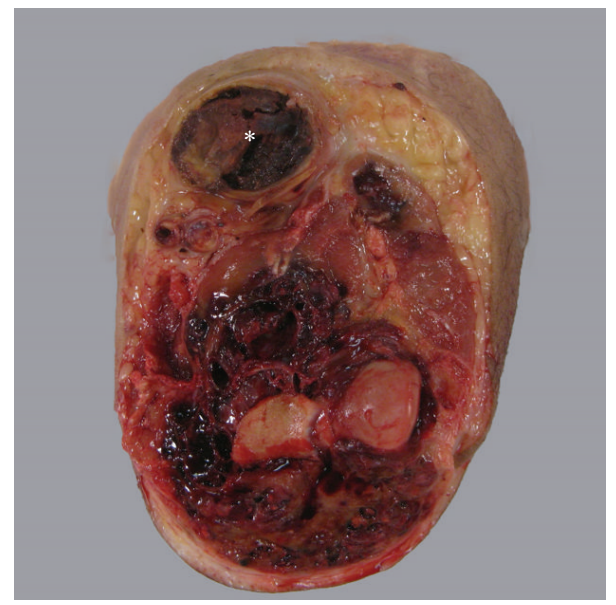

FIGURE 2: Gross examination, amputation specimen. The AVG site (asterisk) in the antecubital region exhibits a dilated space distended with tumor, which radiologically was thought to be thrombus within a pseudoaneurysm. Tumor envelopes the elbow and tracks distally into the forearm.

the forearm. The mass was biopsied to show angiosarcoma, resulting in transhumeral amputation. Six months after the amputation, the patient is alive without evidence of sarcoma.

2.1. Pathology. Initial biopsy of the mass disclosed clusters of malignant epithelioid cells adjacent to poorly formed vascular spaces that showed strong, diffuse expression of vascular markers by immunohistochemistry (Figure 1). Patchy cytokeratin expression paralleled the epithelioid morphology (CK AE1/AE3, CK7, and CK8/18). The subsequent amputation specimen showed tumor at the AVG site (Figure 2). Microscopic examination showed predominantly epithelioid cells with minimal spindle cell morphology and near-absent vasoformation; therefore, a final diagnosis of epithelioid angiosarcoma was rendered. Among the most interesting findings were (1) the presence of tumor juxtaposed to foreign graft material and (2) the finding of tumor tracking along a vessel wall. Two lymph nodes recovered near the resection margin showed metastatic angiosarcoma. The salient histopathologic findings are shown in Figure 3.

2.2. Molecular Findings. FISH analysis for the quantitation of MYC was performed on formalin-fixed, paraffin-embedded tissue sections cut at $5 \mathrm{~mm}$ using commercially available break-apart FISH probe-set for the MYC locus (8q24), with the $5^{\prime}$ probe labeled with Spectrum Red and the $3^{\prime}$ probe labeled with Spectrum Green (Abbott Molecular, Des Plaines, IL, USA). With this probe-set a yellow fusion signal is produced by the juxtaposition of the $5^{\prime}$ and the $3^{\prime}$ probe when the $M Y C$ locus has a normal configuration. Standard laboratory protocols and quality control measures were followed for this study. In each case, 100 interphase nuclei were analyzed in a blinded manner by two technicians (200 total nuclei). Amplification of the MYC locus was defined as the presence of increased number of MYC signals (more than 6) in tumor cells. Clear high-level MYC amplification was seen in large areas, with an average of more than 20 signals per cell (Figure 4).

\section{Discussion}

The finding of $M Y C$ amplification in this case of AVGassociated angiosarcoma expands the spectrum of recognized clinicopathologic subtypes of angiosarcoma bearing this aberration and suggests they share similar pathogenesis. The present case exhibited large regions of clear signal amplification by FISH, consistent with the most commonly observed pattern described in the literature for angiosarcoma [15]. One of the many effects of MYC gene amplification is augmentation of a cell's preexisting gene expression program [20] and promotion of angiogenesis [15]. The presence of MYC amplification in this case does not necessarily exclude a pathogenic role for the synthetic graft material. There is evidence to suggest that the fibrous capsule that develops around foreign material may stimulate carcinogenesis by providing a home-base for macrophages, which secrete cytokines, growth factors, and free radicals that can induce genetic damage, even after fibrosis is complete [14]. Perhaps this mechanism can account for MYC gene aberration in foreign body-associated angiosarcoma. Although the fibrous capsule in this case was fully appreciated, documentation of fibrous encapsulation tends to be inconsistently reported [21].

Alternatively, the graft material may itself directly provoke angiogenesis and subsequent tumorigenesis. Indeed, newer-generation "biopolymers" seek to incorporate bioactive molecules that directly stimulate vascular regeneration [13]. Further studies will be necessary to investigate this and other possible contributing factors, such as immunosuppression. A link to immunosuppression in this case could not be established because the patient was partially managed by an external institution. In any event, the true underlying 


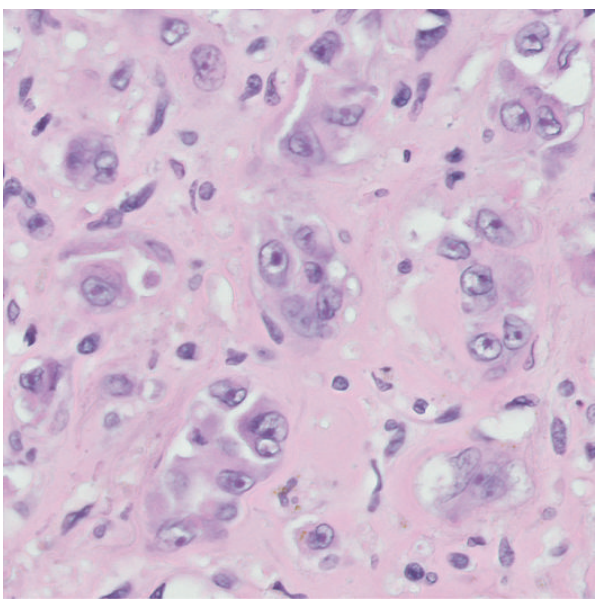

(a)

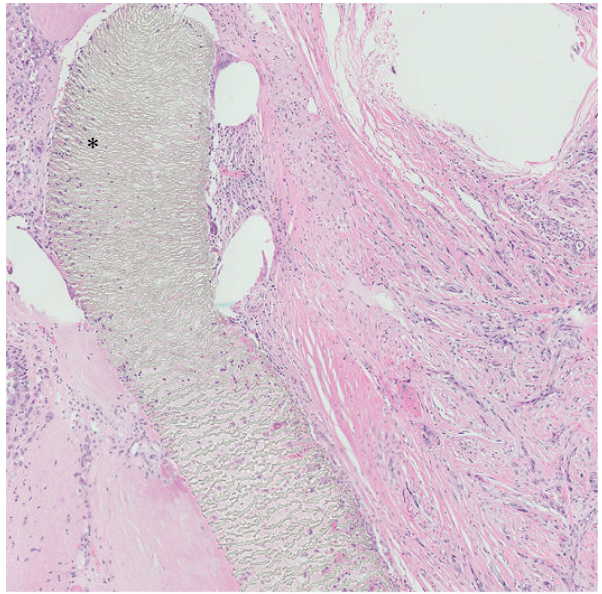

(c)

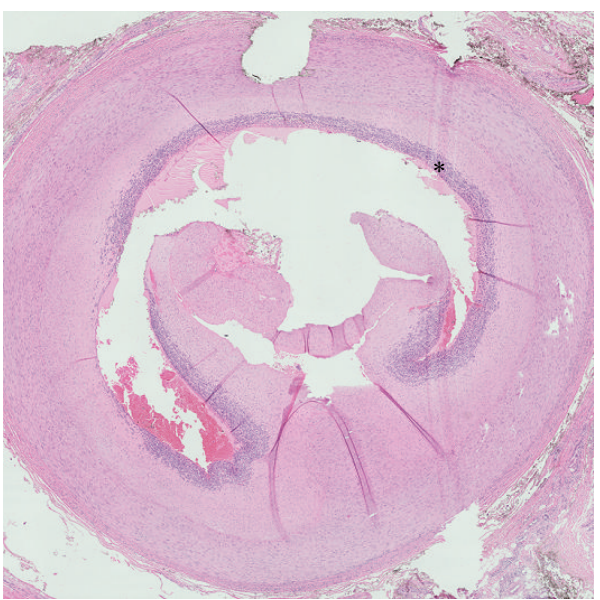

(e)

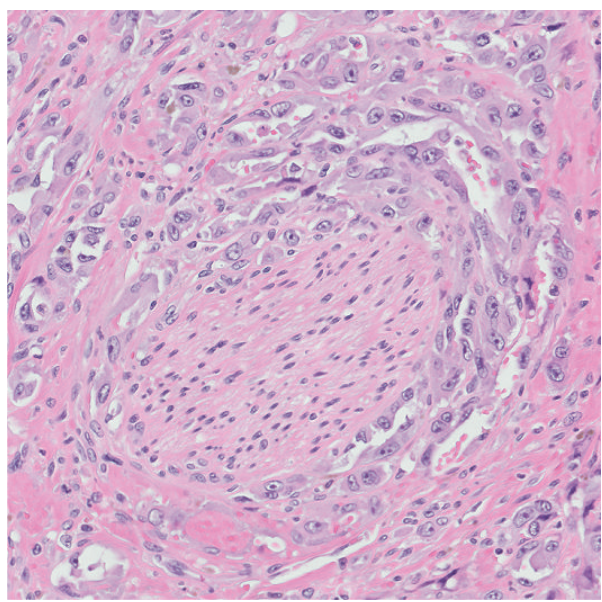

(b)

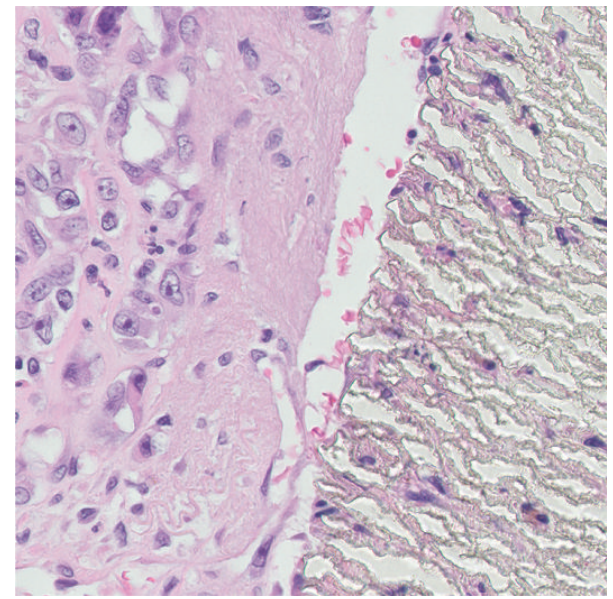

(d)

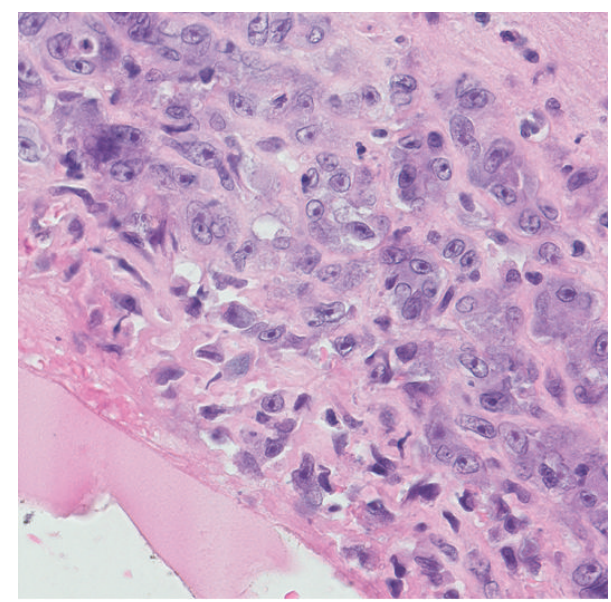

(f)

FIGURE 3: Microscopic examination, amputation specimen. (a) Clusters of malignant epithelioid cells, similar to those seen in the biopsy specimen, are noted (original magnification $\times 400$ ). (b) Perineural invasion can explain the patient's pain symptoms (original magnification $\times 200$ ). (c) Tumor clusters are embedded within the dense fibrous sheath around the graft material; the region marked by the asterisk is shown at higher power in $(\mathrm{d})$ (original magnification $\times 50, \times 400$ ). (e) Tumor cells are seen tracking along the intimal layer of a large artery; the region marked by the asterisk is shown at higher power in (f) (original magnification $\times 10, \times 400$ ). 


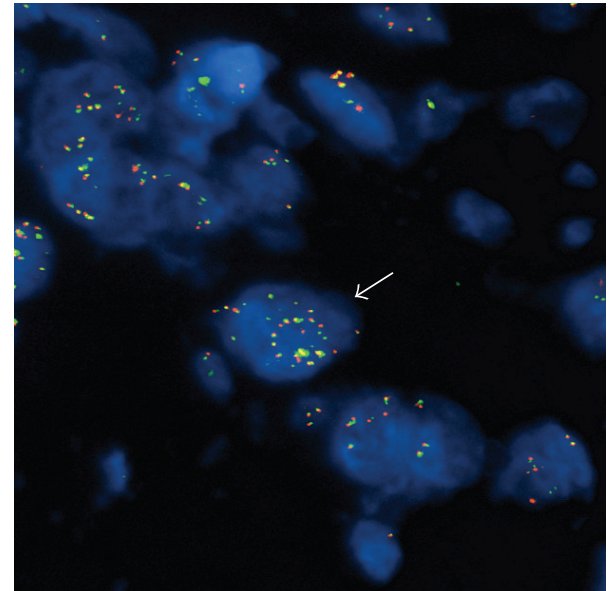

FIGURE 4: FISH results using a MYC break-apart probe. Representative tumor cell (indicated by arrow) is showing an increased number of fusion signals (more than 20), indicative of high-level amplification of the MYC locus.

mechanism for tumorigenesis in this case and in many cases of angiosarcoma likely involves a tangled web of interactions between genetic changes and microenvironment alterations.

\section{Conflict of Interests}

The authors declare that there are no financial disclosures for this study.

\section{References}

[1] R. J. Young, N. J. Brown, M. W. Reed, D. Hughes, and P. J. Woll, "Angiosarcoma," The Lancet Oncology, vol. 11, no. 10, pp. 983991, 2010.

[2] Y. A. Qureshi, D. C. Strauss, K. Thway, C. Fisher, and J. M. Thomas, "Angiosarcoma developing in a non-functioning arteriovenous fistula post-renal transplant," Journal of Surgical Oncology, vol. 101, no. 6, pp. 520-523, 2010.

[3] K. Demey, L.-M. Reyns, and S. Schepers, "Angiosarcoma arising in an arteriovenous fistula in a patient without kidney transplant," Acta Chirurgica Belgica, vol. 114, no. 1, pp. 75-78, 2014.

[4] M. M. Keane and D. N. Carney, "Angiosarcoma arising from a defunctionalized arteriovenous fistula," Journal of Urology, vol. 149, no. 2, pp. 364-365, 1993.

[5] N. R. Parrott, P. D. Scott, A. J. Freemont, and R. W. G. Johnson, "Angiosarcoma in an arteriovenous fistula following successful renal transplantation-a case report," Transplantation, vol. 55, no. 3, pp. 676-677, 1993.

[6] P. J. Conlon Jr., T. Daly, G. Doyle, and M. Carmody, "Angiosarcoma at the site of a ligated arteriovenous fistula in a renal transplant recipient," Nephrology Dialysis Transplantation, vol. 8, no. 3, pp. 259-262, 1993.

[7] B. M. Wehrli, D. L. Janzen, O. Shokeir, B. A. Masri, S. K. Byrne, and J. X. O'Connell, "Epithelioid angiosarcoma arising in a surgically constructed arteriovenous fistula: a rare complication of chronic immunosuppression in the setting of renal transplantation," The American Journal of Surgical Pathology, vol. 22, no. 9, pp. 1154-1159, 1998.
[8] D. Bessis, A. Sotto, P. Roubert, P.-E. Chabrier, G. Mourad, and J.-J. Guilhou, "Endothelin-secreting angiosarcoma occurring at the site of an arteriovenous fistula for haemodialysis in a renal transplant recipient," British Journal of Dermatology, vol. 138, no. 2, pp. 361-363, 1998.

[9] R. Farag, J. A. Schulak, F. W. Abdul-Karim, and J. K. Wasman, "Angiosarcoma arising in an arteriovenous fistula site in a renal transplant patient: a case report and literature review," Clinical Nephrology, vol. 63, no. 5, pp. 408-412, 2005.

[10] J. Chanyaputhipong, D. L. T. Hock, and M. G. Sebastian, "Disseminated angiosarcoma of the dialysis fistula in 2 patients without kidney transplants," American Journal of Kidney Diseases, vol. 57, no. 6, pp. 917-920, 2011.

[11] P. Webster, L. Wujanto, C. Fisher et al., "Malignancies confined to disused arteriovenous fistulae in renal transplant patients: an important differential diagnosis," American Journal of Nephrology, vol. 34, no. 1, pp. 42-48, 2011.

[12] C. D. M. Fletcher, J. A. Bridge, P. C. W. Hogendoorn, and F. Mertens, Eds., WHO Classification of Tumours of Soft Tissue and Bone, IARC, Lyon, France, 2013.

[13] S. Ravi and E. L. Chaikof, "Biomaterials for vascular tissue engineering," Regenerative Medicine, vol. 5, no. 1, pp. 107-120, 2010.

[14] T. G. Moizhess, "Carcinogenesis induced by foreign bodies," Biochemistry, vol. 73, no. 7, pp. 763-775, 2008.

[15] C. Antonescu, "Malignant vascular tumors-an update," Modern Pathology, vol. 27, supplement 1, pp. S30-S38, 2014.

[16] T. Guo, L. Zhang, N.-E. Chang, S. Singer, R. G. Maki, and C. R. Antonescu, "Consistent MYC and FLT4 gene amplification in radiation-induced angiosarcoma but not in other radiationassociated atypical vascular lesions," Genes Chromosomes and Cancer, vol. 50, no. 1, pp. 25-33, 2011.

[17] J. Manner, B. Radlwimmer, P. Hohenberger et al., "MYC high level gene amplification is a distinctive feature of angiosarcomas after irradiation or chronic lymphedema," The American Journal of Pathology, vol. 176, no. 1, pp. 34-39, 2010.

[18] S. L. J. Verbeke, D. de Jong, F. Bertoni et al., "Array CGH analysis identifies two distinct subgroups of primary angiosarcoma of bone," Genes Chromosomes and Cancer, vol. 54, no. 2, pp. 72-81, 2014.

[19] W. Shon, W. R. Sukov, S. M. Jenkins, and A. L. Folpe, "MYC amplification and overexpression in primary cutaneous angiosarcoma: a fluorescence in-situ hybridization and immunohistochemical study," Modern Pathology, vol. 27, no. 4, pp. 509-515, 2014.

[20] C. Y. Lin, J. Lovén, P. B. Rahl et al., "Transcriptional amplification in tumor cells with elevated c-Myc," Cell, vol. 151, no. 1, pp. 56-67, 2012.

[21] T. A. Jennings, L. Peterson, C. A. Axiotis, G. E. Friedlaender, R. A. Cooke, and J. Rosai, "Angiosarcoma associated with foreign body material: a report of three cases," Cancer, vol. 62, no. 11, pp. 2436-2444, 1988. 


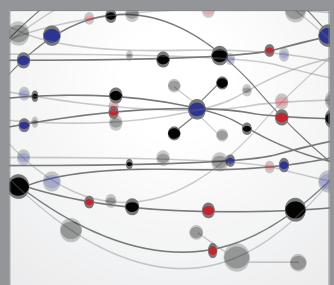

The Scientific World Journal
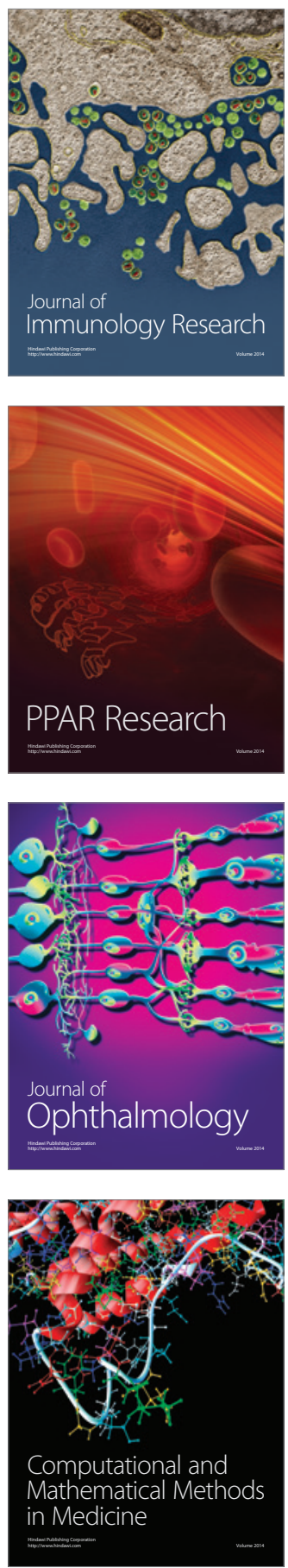

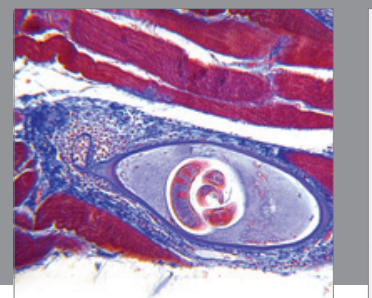

Gastroenterology

Research and Practice
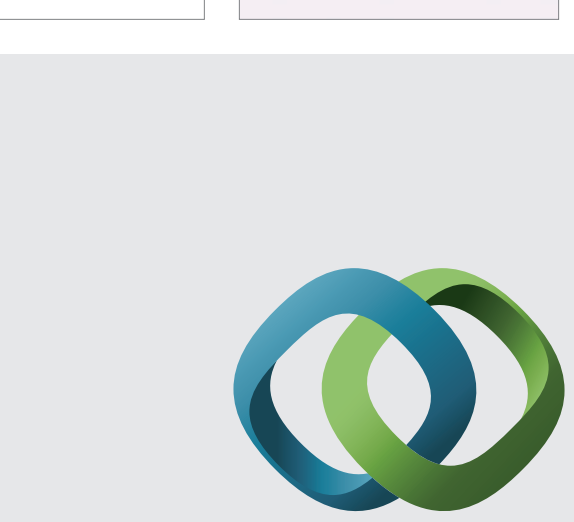

\section{Hindawi}

Submit your manuscripts at

http://www.hindawi.com
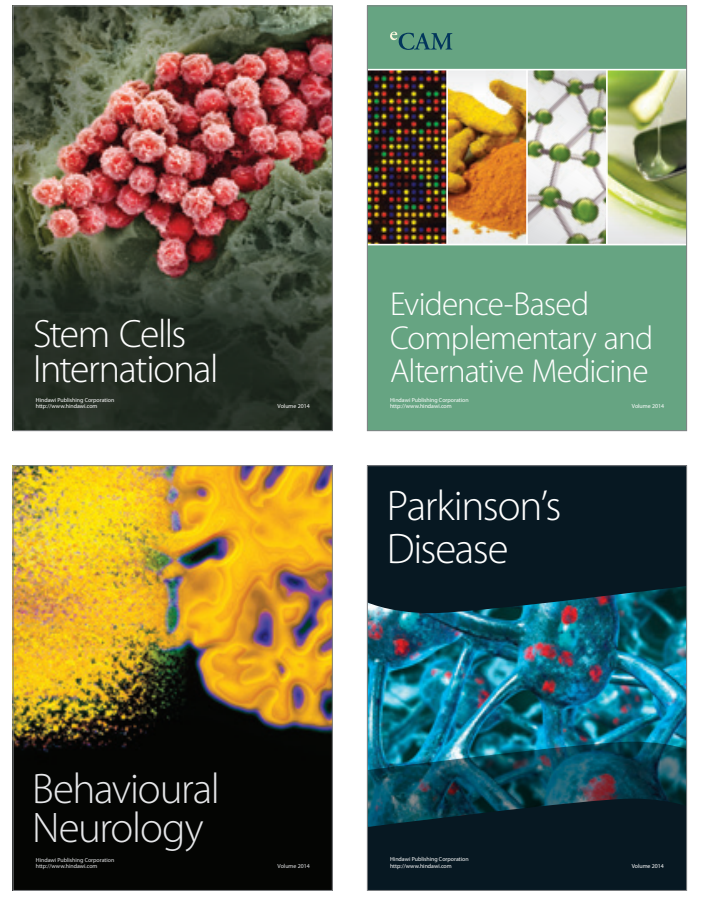
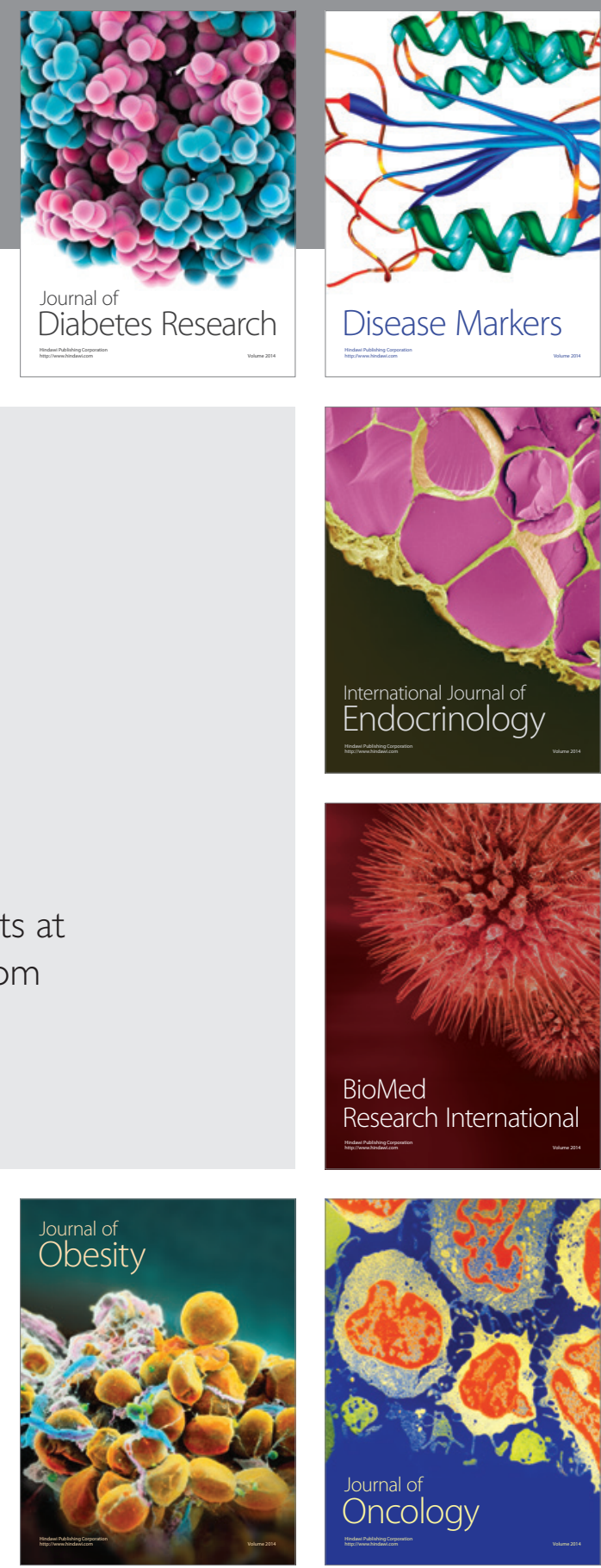

Disease Markers
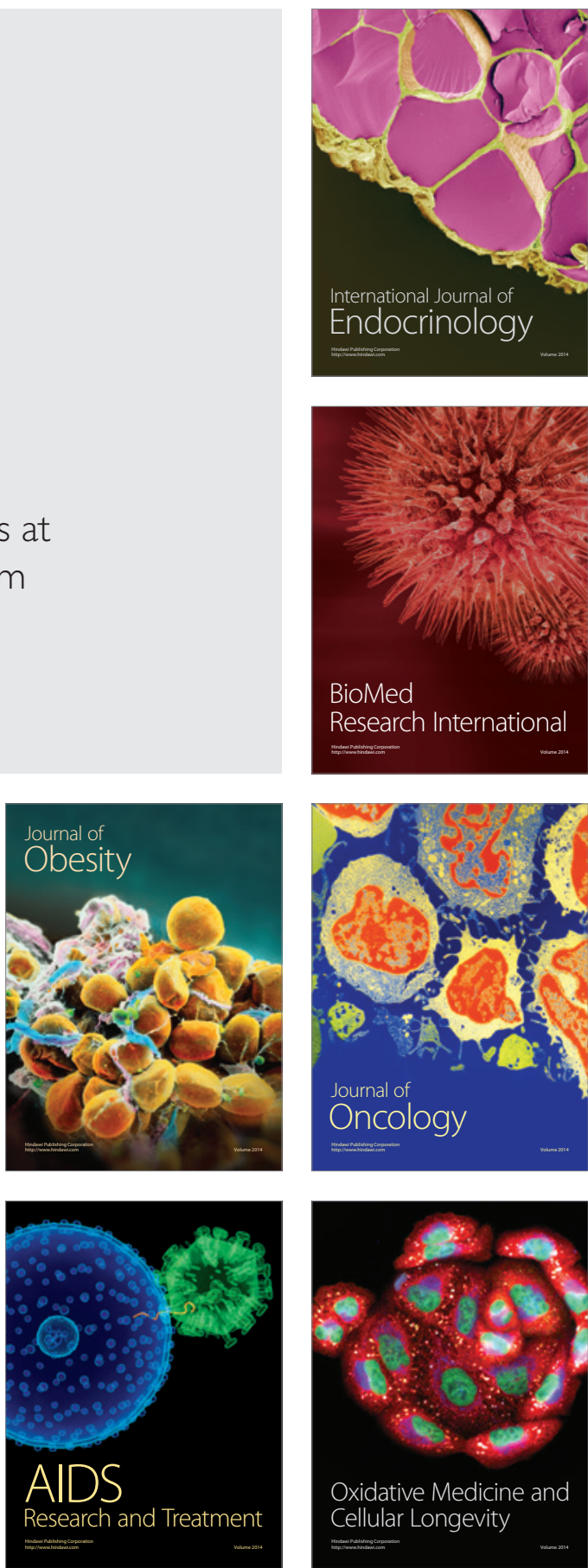